\title{
Extent of resection is important across glioblastoma molecular subtypes
}

Glioblastoma is classified according to $I D H$ mutational status, with $I D H$-wildtype disease having a substantially worse prognosis than $I D H$-mutant disease. Surgery is a mainstay treatment for both forms of glioblastoma, although the influence of extent of resection has been unclear. Now, new data provide important insights across molecular subtypes.

The new data come from a retrospective multicentre study involving 761 patients. Cox proportional hazards-regression modelling revealed that patients with $I D H$-wild-type and those with $I D H$-mutant glioblastoma both benefit from a greater percentage resection of not only contrast-enhancing (CE) tumour visible on T1-weighted MRI, but also non-contrast-enhancing (NCE) tumour visible on T2-weighted or FLAIR MRI, regardless of MGMT promoter-methylation status.

Among patients with $I D H$-wild-type disease treated with adjuvant temozolomide, only younger patients ( $<65$ years old) benefitted from maximal resection of NCE in addition to CE tumour. In particular, a subgroup with a median $100 \%$ and $92 \%$ resection of CE and NCE tumour, respectively (resulting in a residual NCE tumour burden of $<5.4 \mathrm{ml}$ ), had a median overall survival (OS) of 31.7 months. By contrast, those with a median $98 \%$ resection of CE tumour but $>5.4 \mathrm{ml}$ of residual NCE tumour (median 54\% NCE tumour resection) had a median OS of 17.9 months. Notably, the OS of the former subgroup was similar to that of patients with $I D H$-mutant disease over the first 2-3 years, but decreased much more rapidly after this point - the median OS was 78.4 months in the IDH-mutant group.

These data will enable better prognostication and provide clarification of the surgical strategy for glioblastoma. Maximal CE and NCE tumour resection is the goal in younger patients, whereas complete resection of CE tumour only might be appropriate in older patients.

\section{David Killock}

ORIGINAL ARTICLE Molinaro, A. M. et al.JAMA Oncol. https://doi.org/10.1001/jamaoncol.2019.6143 (2020)

\section{Intestinal microbiota predict HSCT outcome}

Allogeneic haematopoietic stem cell transplantation (HSCT) provides the possibility of cure for patients with haematological malignancies. Nonetheless, the high risks of potentially lethal graft-versus-host disease (GVHD), infections and other adverse outcomes limit the effectiveness of this intervention and are difficult to predict in advance. Now, data from a large-cohort observational study provide prospective evidence of the intestinal microbiota as a predictor of mortality in patients undergoing HSCT.

A total of 1,362 patients were enrolled at four centres; data from patients enrolled at MSKCC (cohort 1) and in other locations (cohort 2) were analysed separately. Baseline stool samples were obtained from patients undergoing HSCT for haematological malignancies (predominantly acute myeloid leukaemia), within 30 days of treatment. Weekly stool samples were obtained over the 3 weeks after HSCT. Each sample was assessed using 16S RNA sequencing.

A more diverse intestinal microbiota after HSCT (defined as above the median inverse Simpson diversity index) was associated with a lower risk of death in cohort 1 (HR $0.71 ; 95 \% \mathrm{Cl} 0.55-0.92)$ and in cohort 2 (HR 0.49; $95 \% \mathrm{Cl}, 0.27-0.90$ ). Comparisons of post-HSCT and pre-HSCT intestinal microbiota diversity revealed a lower risk of death (HR $0.41,95 \% \mathrm{Cl} 0.24-0.71$ ) and transplantation-related death ( $\mathrm{HR} 0.44,95 \% \mathrm{Cl}$ $0.22-0.87$ ) in patients with higher pre-HSCT diversity when assessed as a continuous variable in cohort 1 . These observations were not validated in cohort 2 , although survival data were immature at the cutoff used. A risk score was compiled based upon common taxonomic features of the pre-HSCT microbiota of patients in cohort 1, and this score was predictive of risk of death in cohort 2 (HR 1.39, 95\% Cl 1.02-1.91).

These findings demonstrate that a more diverse intestinal microbiota is associated with a lower risk of mortality following HSCT. Associations between specific taxa and outcomes will require further validation.

Peter Sidaway

ORIGINAL ARTICLE Peled, J. U. et al. Microbiota as predictor of mortality in allogeneic hematopoietic-cell transplantation. N. Engl.J. Med. 382, 822-834 (2020)

\section{New standard for localized UTUC}

Upper tract urothelial carcinoma (UTUC; transitional cell carcinoma of the ureter or renal pelvis) has a worse stage-for-stage prognosis than the related disease, urothelial carcinoma of the bladder. UTUC is muscleinvasive or locally advanced at diagnosis in $>50 \%$ of patients and a similar proportion ultimately die of the disease. Drawing on the clinical benefits achieved with platinum-based chemotherapy for bladder cancer, Birtle et al. initiated the phase III POUT trial of adjuvant chemotherapy for patients with muscleinvasive or lymph node-positive UTUC. Now, data from POUT support this treatment approach as a new standard of care.

Enrolment for the POUT trial was stopped early after a preplanned interim analysis of data from 261 patients revealed a diseasefree survival (DFS) benefit of chemotherapy following nephroureterectomy and resection of any known abnormal lymph nodes. Among 131 patients who received up to four cycles of gemcitabine plus carboplatin or cisplatin, estimated 3-year DFS was $71 \%$, compared with $46 \%$ in 129 patients who underwent standard surveillance only (HR 0.45, 95\% Cl 0.30-0.68; $P=0.0001$ ); 3-year metastasis-free survival was also improved ( $71 \%$ versus $53 \%$; $\mathrm{HR} 0.48,95 \% \mathrm{Cl}$ $0.31-0.74 ; P=0.0007)$. These benefits were largely unchanged in multivariate analyses.

Unsurprisingly, chemotherapy significantly increased the risk of toxicities: grade $>3$ adverse events, mostly decreased neutrophil and/or platelet counts, nausea and vomiting or febrile neutropenia, occurred in $44 \%$ of patients, compared with only $4 \%$ of the surveillance group $(P<0.0001)$. Importantly, no treatment-related deaths occurred. Quality of life questionnaires were completed by at least $70 \%$ of patients in each group and revealed declines in mean overall global health status scores during and immediately after chemotherapy, which resolved by 12 months.

UTUC is a rare disease affecting $\sim 2$ people per 100,000. Thus, Birtle et al. should be commended for performing what is almost certainly the first dedicated phase III study of perioperative therapy for UTUC and the largest-cohort randomized controlled trial in this disease to date. Longer-term results of POUT, including overall survival data, are awaited.

David Killock

ORIGINAL ARTICLE Birtle, A. et al. Adjuvant chemotherapy in upper tract urothelial carcinoma (the POUT trial): a phase 3 , open-label, randomised controlled trial. Lancet https://doi.org/ 10.1016/S0140-6736(20)30415-3(2020) 